\title{
Convolutions of the bi-periodic Fibonacci numbers
}

\author{
Takao Komatsu ${ }^{1}$ (D), José L. Ramírez ${ }^{* 2}$ (D) \\ ${ }^{1}$ Department of Mathematics, School of Science, Zhejiang Sci-Tech University, Hangzhou, 310018, China \\ ${ }^{2}$ Departamento de Matemáticas, Universidad Nacional de Colombia, Bogotá, Colombia
}

\begin{abstract}
Let $q_{n}$ be the bi-periodic Fibonacci numbers, defined by $q_{n}=c(n) q_{n-1}+q_{n-2}(n \geq 2)$ with $q_{0}=0$ and $q_{1}=1$, where $c(n)=a$ if $n$ is even, $c(n)=b$ if $n$ is odd, where $a$ and $b$ are nonzero real numbers. When $c(n)=a=b=1, q_{n}=F_{n}$ are Fibonacci numbers. In this paper, the convolution identities of order 2, 3 and 4 for the bi-periodic Fibonacci numbers $q_{n}$ are given with binomial (or multinomial) coefficients, by using the symmetric formulas.
\end{abstract}

Mathematics Subject Classification (2010). 11B39, 05A15, 05A19

Keywords. bi-periodic Fibonacci numbers, convolutions, symmetric formulas

\section{Introduction}

Convolution identities for various types of numbers (or polynomials) have been studied, with or without binomial (or multinomial) coefficients, including Bernoulli, Euler, Genocchi, Cauchy, Stirling and balancing numbers (cf. [1-3,6,9,10,15,16,19]). A typical formula is due to Euler, given by

$$
\sum_{k=0}^{n}\left(\begin{array}{l}
n \\
k
\end{array}\right) \mathcal{B}_{k} \mathcal{B}_{n-k}=-n \mathcal{B}_{n-1}-(n-1) \mathcal{B}_{n} \quad(n \geq 0),
$$

where $\mathcal{B}_{n}$ are Bernoulli numbers, defined by

$$
\frac{x}{e^{x}-1}=\sum_{n=0}^{\infty} \mathcal{B}_{n} \frac{x^{n}}{n !} \quad(|x|<2 \pi) .
$$

On the other hand, many kinds of generalizations of Fibonacci numbers have been presented in the literature. A typical one is a generalized Fibonacci sequence $\left\{W_{n}\right\}_{n=0}^{\infty}$, defined by $W_{n}=p W_{n-1}+q W_{n-2}(n \geq 2)$ with $W_{0}=a$ and $W_{1}=b$. In [5] some new identities involving differences of products of generalized Fibonacci numbers are shown. One of different types is the bi-periodic Fibonacci sequence [7]. For any two nonzero real numbers $a$ and $b$, the bi-periodic Fibonacci sequence, say $\left\{q_{n}\right\}_{n=0}^{\infty}$, is determined by:

$$
q_{0}=0, \quad q_{1}=1, \quad q_{n}=\left\{\begin{array}{lll}
a q_{n-1}+q_{n-2}, & \text { if } n \equiv 0 \quad(\bmod 2) ; \\
b q_{n-1}+q_{n-2}, & \text { if } n \equiv 1 \quad(\bmod 2) ;
\end{array} \quad n \geqslant 2 .\right.
$$

\footnotetext{
*Corresponding Author.

Email addresses: komatsu@whu.edu.cn (T. Komatsu), jlramirezr@unal.edu.co (J.L. Ramírez)

Received: 16.08.2017; Accepted: 22.01.2019
} 
When $a=b=1, q_{n}=F_{n}$ are Fibonacci numbers. The explicit expression of the biperiodic Fibonacci numbers can be expressed explicitly as

$$
\begin{aligned}
q_{2 n} & =a \sum_{k=0}^{n-1}\left(\begin{array}{c}
2 n-k-1 \\
k
\end{array}\right)(a b)^{n-k-1}, \\
q_{2 n+1} & =\sum_{k=0}^{n}\left(\begin{array}{c}
2 n-k \\
k
\end{array}\right)(a b)^{n-k} .
\end{aligned}
$$

Moreover, the ordinary generating function of the bi-periodic Fibonacci numbers is given by

$$
F(x):=\sum_{n=0}^{\infty} q_{n} x^{n}=\frac{x\left(1+a x-x^{2}\right)}{1-(a b+2) x^{2}+x^{4}} .
$$

For more properties about this sequence see for example $[4,7,8,18,20]$.

Recently, in [13], the convolution identities of two Fibonacci numbers $F_{n}$ are explicitly given:

$$
\sum_{k=0}^{n} F_{k} F_{n-k}=\sum_{m=0}^{n-1} m F_{m} \cos \frac{(n-m-1) \pi}{2}
$$

as special cases of higher-order identities. In [14], this result is generalized by using a more general form:

$$
\frac{F_{r}+(-1)^{r} F_{k-r} x}{1-L_{k} x+(-1)^{k} x^{2}}=\sum_{n=0}^{\infty} F_{k n+r} x^{n},
$$

with $k>r \geq 0$, where $L_{n}$ are Lucas numbers. In [11,12,17], convolution identities for Fibonacci numbers are generalized as Tribonacci numbers and Tetranacci numbers. In particular, in $[12,17]$, symmetric formulas are used to yield the results.

In this paper, motivated by the previous results, the convolution identities for the biperiodic Fibonacci numbers $q_{n}$ are given with binomial (or multinomial) coefficients. In [15] the so-called exponential generating functions of generalized Fibonacci-type numbers $u_{n}$ and Lucas-type numbers $v_{n}$ are considered:

$$
\frac{e^{\alpha x}-e^{\beta x}}{\sqrt{a^{2}+4 b}}=\sum_{n=0}^{\infty} u_{n} \frac{x^{n}}{n !} \quad \text { and } \quad e^{\alpha x}+e^{\beta x}=\sum_{n=0}^{\infty} v_{n} \frac{x^{n}}{n !} .
$$

Here, $u_{n}=a u_{n-1}+b u_{n-2}(n \geq 2)$ with $u_{0}=0$ and $u_{1}=1$, and $v_{n}=a v_{n-1}+b v_{n-2}(n \geq 2)$ with $v_{0}=2$ and $v_{1}=a$. $\alpha$ and $\beta$ are the roots of the quadratic equation $x^{2}-a x-b=0$, given by

$$
\alpha=\frac{a+\sqrt{a^{2}+4 b}}{2} \text { and } \beta=\frac{a-\sqrt{a^{2}+4 b}}{2} .
$$

Then the higher-order convolution identities with multinomial coefficients

$$
\sum_{\substack{k_{1}+\cdots+k_{r}=n \\
k_{1}, \ldots, k_{r} \geq 0}}\left(\begin{array}{c}
n \\
k_{1}, \ldots, k_{r}
\end{array}\right) u_{k_{1}} \cdots u_{k_{r}} \text { and } \sum_{\substack{k_{1}+\cdots+k_{r}=n \\
k_{1}, \ldots, k_{r} \geq 0}}\left(\begin{array}{c}
n \\
k_{1}, \ldots, k_{r}
\end{array}\right) v_{k_{1}} \cdots v_{k_{r}}
$$

are given in the linear combinations of $u_{n}$ and $v_{n}$. We consider this kind of convolution identities for bi-periodic Fibonacci numbers.

This paper is organized as follows. In Section 2, convolution identities for two biperiodic Fibonacci numbers with binomial coefficients are shown. In Section 3, convolution identities for three and four bi-periodic Fibonacci numbers with multinomial coefficients are shown. The main tools are symmetric formulas which are often used in $[12,17]$. 


\section{Convolution identities with binomial coefficients}

Consider the exponential generating function

$$
f(x):=\sum_{n=0}^{\infty} q_{n} \frac{x^{n}}{n !} .
$$

We introduce two supplementary functions

$$
f_{1}(x)=\sum_{n=0}^{\infty} q_{2 n} \frac{x^{2 n}}{(2 n) !} \quad \text { and } \quad f_{2}(x)=\sum_{n=0}^{\infty} q_{2 n+1} \frac{x^{2 n+1}}{(2 n+1) !}
$$

so that $f(x)=f_{1}(x)+f_{2}(x)$. By using the recurrence relations (1.1), we have the system of the differential equations:

$$
\begin{aligned}
& f_{1}^{\prime \prime}(x)-a f_{2}^{\prime}(x)-f_{1}(x)=0 \\
& f_{2}^{\prime \prime}(x)-b f_{1}^{\prime}(x)-f_{2}(x)=0 .
\end{aligned}
$$

Therefore, we get two 4 -th order differential equations:

$$
\begin{aligned}
f_{1}^{(4)}(x)-(a b+2) f_{1}^{\prime \prime}(x)+f_{1}(x) & =0 \\
\left.f_{2}^{(4)}(x)-(a b+2) f_{2}^{\prime \prime}(x)+f_{2}(x)\right) & =0 .
\end{aligned}
$$

Since the roots of $x^{4}-(a b+2) x^{2}+1=0$ are given by

$$
\pm \alpha= \pm \sqrt{\frac{a b+2+\sqrt{a b(a b+4)}}{2}} \text { and } \pm \beta= \pm \sqrt{\frac{a b+2-\sqrt{a b(a b+4)}}{2}}
$$

the generating function $f_{1}(x)$ can be expressed as

$$
f_{1}(x)=c_{1} e^{\alpha x}+c_{2} e^{-\alpha x}+c_{3} e^{\beta x}+c_{4} e^{-\beta x},
$$

where

$$
\begin{aligned}
c_{1}+c_{2}+c_{3}+c_{4} & =q_{0}=0, \\
c_{1} \alpha-c_{2} \alpha+c_{3} \beta-c_{4} \beta & =0, \\
c_{1} \alpha^{2}+c_{2} \alpha^{2}+c_{3} \beta^{2}+c_{4} \beta^{2} & =q_{2}=a, \\
c_{1} \alpha^{3}-c_{2} \alpha^{3}+c_{3} \beta^{3}-c_{4} \beta^{3} & =0 .
\end{aligned}
$$

Solving this system, we get

$$
c_{1}=c_{2}=\frac{a}{2 \sqrt{a b(a b+4)}} \quad \text { and } \quad c_{3}=c_{4}=-\frac{a}{2 \sqrt{a b(a b+4)}} .
$$

Note that $\alpha \beta=1, \alpha^{2}+\beta^{2}=a b+2$ and $\alpha^{2}-\beta^{2}=\sqrt{a b(a b+4)}$.

Similarly, the generating function $f_{2}(x)$ can be expressed as

$$
f_{2}(x)=d_{1} e^{\alpha x}+d_{2} e^{-\alpha x}+d_{3} e^{\beta x}+d_{4} e^{-\beta x},
$$

where

$$
\begin{aligned}
d_{1}+d_{2}+d_{3}+d_{4} & =0 \\
d_{1} \alpha-d_{2} \alpha+d_{3} \beta-d_{4} \beta & =q_{1}=1, \\
d_{1} \alpha^{2}+d_{2} \alpha^{2}+d_{3} \beta^{2}+d_{4} \beta^{2} & =0 \\
d_{1} \alpha^{3}-d_{2} \alpha^{3}+d_{3} \beta^{3}-d_{4} \beta^{3} & =q_{3}=a b+1 .
\end{aligned}
$$


Solving this system, we get

$$
\begin{aligned}
& d_{1}=-d_{2}=\frac{a b+4+\sqrt{a b(a b+4)}}{2 \sqrt{2}(a b+4) \sqrt{a b+2+\sqrt{a b(a b+4)}}}, \\
& d_{3}=-d_{4}=\frac{a b+4-\sqrt{a b(a b+4)}}{2 \sqrt{2}(a b+4) \sqrt{a b+2-\sqrt{a b(a b+4)}}} .
\end{aligned}
$$

Therefore, we obtain that

$$
f(x)=r_{1} e^{\alpha x}+r_{2} e^{-\alpha x}+r_{3} e^{\beta x}+r_{4} e^{-\beta x},
$$

where

$$
\begin{aligned}
& r_{1}=c_{1}+d_{1}=\frac{a}{2 \sqrt{a b(a b+4)}}+\frac{a b+4+\sqrt{a b(a b+4)}}{2 \sqrt{2}(a b+4) \sqrt{a b+2+\sqrt{a b(a b+4)}}}, \\
& r_{2}=c_{2}+d_{2}=\frac{a}{2 \sqrt{a b(a b+4)}}-\frac{a b+4+\sqrt{a b(a b+4)}}{2 \sqrt{2}(a b+4) \sqrt{a b+2+\sqrt{a b(a b+4)}}}, \\
& r_{3}=c_{3}+d_{3}=-\frac{a}{2 \sqrt{a b(a b+4)}}+\frac{a b+4-\sqrt{a b(a b+4)}}{2 \sqrt{2}(a b+4) \sqrt{a b+2-\sqrt{a b(a b+4)}}}, \\
& r_{4}=c_{4}+d_{4}=-\frac{a}{2 \sqrt{a b(a b+4)}}-\frac{a b+4-\sqrt{a b(a b+4)}}{2 \sqrt{2}(a b+4) \sqrt{a b+2-\sqrt{a b(a b+4)}}} .
\end{aligned}
$$

Now, we shall consider the sum of the product of two bi-periodic Fibonacci numbers. We need three Lemmas to get the main result.

\section{Lemma 2.1.}

$$
r_{1}^{2} e^{2 \alpha x}+r_{2}^{2} e^{-2 \alpha x}+r_{3}^{2} e^{2 \beta x}+r_{4}^{2} e^{-2 \beta x}=\frac{1}{b(a b+4)} \sum_{n=0}^{\infty} Q_{n} \frac{x^{n}}{n !},
$$

where $Q_{n}$ are numbers, satisfying for $n \geq 1$

$$
\begin{aligned}
Q_{2 n} & =(a+b) Q_{2 n-1}+4 Q_{2 n-2}, \\
Q_{2 n+1} & =\frac{4 a b}{a+b} Q_{2 n}+4 Q_{2 n-1},
\end{aligned}
$$

with $Q_{0}=a+b$ and $Q_{1}=2 a b$.

Remark 2.2. We have explicit expressions: for $n \geq 0$

$$
\begin{aligned}
Q_{2 n} & =2^{2 n-1}(a+b) \sum_{k=0}^{n} \frac{2 n}{2 n-k}\left(\begin{array}{c}
2 n-k \\
k
\end{array}\right)(a b)^{n-k}, \\
Q_{2 n+1} & =2^{2 n+1} a b \sum_{k=0}^{n} \frac{2 n+1}{2 n-k+1}\left(\begin{array}{c}
2 n-k+1 \\
k
\end{array}\right)(a b)^{n-k} .
\end{aligned}
$$

Proof of Lemma 2.1. Assume that the exponential generating function

$$
r_{1}^{2} e^{2 \alpha x}+r_{2}^{2} e^{-2 \alpha x}+r_{3}^{2} e^{2 \beta x}+r_{4}^{2} e^{-2 \beta x}
$$

determines the sequence $\left\{\widehat{Q}_{n}\right\}_{n=0}^{\infty}$. By $\alpha \beta=1$ and $\alpha^{2}+\beta^{2}=a b+2$, we have the characteristic equation

$$
\begin{aligned}
& (x+2 \alpha)(x-2 \alpha)(x+2 \beta)(x-2 \beta) \\
& =x^{4}-4\left(\alpha^{2}+\beta^{2}\right) x^{2}+16 \alpha^{2} \beta^{2} \\
& =x^{4}-4(a b+2) x^{2}+16 .
\end{aligned}
$$


Thus, $\widehat{Q}_{n}$ satisfies the recurrence relation

$$
\widehat{Q}_{n}=4(a b+2) \widehat{Q}_{n-2}-16 \widehat{Q}_{n-4} \quad(n \geq 4) .
$$

Now, we get that

$$
\begin{aligned}
& \widehat{Q}_{0}=r_{1}^{2}+r_{2}^{2}+r_{3}^{2}+r_{4}^{2}=\frac{a+b}{b(a b+4)}, \\
& \widehat{Q}_{1}=2 r_{1}^{2} \alpha-2 r_{2}^{2} \alpha+2 r_{3}^{2} \beta-2 r_{4}^{2} \beta=\frac{2 a}{a b+4}, \\
& \widehat{Q}_{2}=4 r_{1}^{2} \alpha^{2}+4 r_{2}^{2} \alpha^{2}+4 r_{3}^{2} \beta^{2}+4 r_{4}^{2} \beta^{2}=\frac{2(a+b)(a b+2)}{b(a b+4)}, \\
& \widehat{Q}_{3}=8 r_{1}^{2} \alpha^{3}-8 r_{2}^{2} \alpha^{3}+8 r_{3}^{2} \beta^{3}-8 r_{4}^{2} \beta^{3}=\frac{8 a(a b+3)}{a b+4} .
\end{aligned}
$$

Using the recurrence relation (2.5), by induction, we have the recurrence relations: for $n \geq 1$

$$
\begin{aligned}
\widehat{Q}_{2 n} & =(a+b) \widehat{Q}_{2 n-1}+4 \widehat{Q}_{2 n-2}, \\
\widehat{Q}_{2 n+1} & =\frac{4 a b}{a+b} \widehat{Q}_{2 n}+4 \widehat{Q}_{2 n-1} .
\end{aligned}
$$

Putting $Q_{n}=b(a b+4) \widehat{Q}_{n}$, we get the desired result.

\section{Lemma 2.3.}

$$
r_{1} r_{3} e^{(\alpha+\beta) x}+r_{2} r_{4} e^{-(\alpha+\beta) x}+r_{1} r_{4} e^{(\alpha-\beta) x}+r_{2} r_{3} e^{-(\alpha-\beta) x}=-\frac{1}{b(a b+4)} \sum_{n=0}^{\infty} Q Q_{n} \frac{x^{n}}{n !},
$$

where for $n \geq 0$

$$
\begin{aligned}
Q Q_{2 n} & =\frac{a-b}{2}\left((a b+4)^{n}-(a b)^{n}\right)+a^{n+1} b^{n}, \\
Q Q_{2 n+1} & =(a b)^{n+1} .
\end{aligned}
$$

Proof. Assume that the exponential generating function

$$
r_{1} r_{3} e^{(\alpha+\beta) x}+r_{2} r_{4} e^{-(\alpha+\beta) x}+r_{1} r_{4} e^{(\alpha-\beta) x}+r_{2} r_{3} e^{-(\alpha-\beta) x}
$$

determines the sequence $\left\{\widehat{Q Q}_{n}\right\}_{n=0}^{\infty}$. By $\alpha^{2}+\beta^{2}=a b+2$ and $\alpha^{2}-\beta^{2}=\sqrt{a b(a b+4)}$, we have the characteristic equation

$$
\begin{aligned}
& (x+(\alpha+\beta))(x-(\alpha+\beta))(x+(\alpha-\beta))(x-(\alpha-\beta)) \\
& =x^{4}-2\left(\alpha^{2}+\beta^{2}\right) x^{2}+\left(\alpha^{2}-\beta^{2}\right)^{2} \\
& =x^{4}-2(a b+2) x^{2}+(a b)(a b+4) .
\end{aligned}
$$

Thus, $\widehat{Q Q}_{n}$ satisfies the recurrence relation

$$
\widehat{Q Q}_{n}=2(a b+2) \widehat{Q Q}_{n-2}-(a b)(a b+4) \widehat{Q Q}_{n-4} \quad(n \geq 4) .
$$


Now, we get that

$$
\begin{aligned}
& \widehat{Q Q}_{0}=r_{1} r_{3}+r_{2} r_{4}+r_{1} r_{4}+r_{2} r_{3}=-\frac{a}{b(a b+4)}, \\
& \widehat{Q Q}_{1}=r_{1} r_{3}(\alpha+\beta)-r_{2} r_{4}(\alpha+\beta)+r_{1} r_{4}(\alpha-\beta)-r_{2} r_{3}(\alpha-\beta)=-\frac{a}{a b+4}, \\
& \widehat{Q Q}_{2}=r_{1} r_{3}(\alpha+\beta)^{2}+r_{2} r_{4}(\alpha+\beta)^{2}+r_{1} r_{4}(\alpha-\beta)^{2}+r_{2} r_{3}(\alpha-\beta)^{2}=-\frac{2(a-b)+a^{2} b}{b(a b+4)}, \\
& \widehat{Q Q}_{3}=r_{1} r_{3}(\alpha+\beta)^{3}-r_{2} r_{4}(\alpha+\beta)^{3}+r_{1} r_{4}(\alpha-\beta)^{3}-r_{2} r_{3}(\alpha-\beta)^{3}=-\frac{a^{2} b}{a b+4} .
\end{aligned}
$$

Using the recurrence relation (2.6), by induction, we have for $n \geq 0$

$$
\begin{aligned}
\widehat{Q Q}_{2 n} & =\frac{(b-a)(a b+4)^{n}-(a+b)(a b)^{n}}{2 b(a b+4)}, \\
\widehat{Q Q}_{2 n+1} & =-\frac{a^{n+1} b^{n}}{a b+4} .
\end{aligned}
$$

Putting $Q Q_{n}=-b(a b+4) \widehat{Q Q}_{n}$, we get the desired result.

\section{Lemma 2.4.}

$$
r_{1} r_{2}+r_{3} r_{4}=\frac{a-b}{2 b(a b+4)}
$$

Proof. Since

we get the result.

$$
r_{1} r_{2}=r_{3} r_{4}=\frac{a}{4 b(a b+4)}-\frac{1}{4(a b+4)}=\frac{a-b}{4 b(a b+4)},
$$

Theorem 2.5. For $n \geq 1$, we have

$$
\sum_{k=0}^{n}\left(\begin{array}{l}
n \\
k
\end{array}\right) q_{k} q_{n-k}=\frac{Q_{n}-2 Q Q_{n}}{b(a b+4)}
$$

Proof. Since $(\hat{a}+\hat{b}+\hat{c}+\hat{d})^{2}=\left(\hat{a}^{2}+\hat{b}^{2}+\hat{c}^{2}+\hat{d}^{2}\right)+2(\hat{a} \hat{b}+\hat{a} \hat{c}+\hat{a} \hat{d}+\hat{b} \hat{c}+\hat{b} \hat{d}+\hat{c} \hat{d})$, by Lemmas 2.1, 2.3 and 2.4 we have

$$
\begin{aligned}
( & \left.r_{1} e^{\alpha x}+r_{2} e^{-\alpha x}+r_{3} e^{\beta x}+r_{4} e^{-\beta x}\right)^{2} \\
= & \left(r_{1}^{2} e^{2 \alpha x}+r_{2}^{2} e^{-2 \alpha x}+r_{3}^{2} e^{2 \beta x}+r_{4}^{2} e^{-2 \beta x}\right) \\
& +2\left(r_{1} r_{3} e^{(\alpha+\beta) x}+r_{2} r_{4} e^{-(\alpha+\beta) x}+r_{1} r_{4} e^{(\alpha-\beta) x}+r_{2} r_{3} e^{-(\alpha-\beta) x}\right) \\
& +2\left(r_{1} r_{2}+r_{3} r_{4}\right) \\
= & \frac{1}{b(a b+4)} \sum_{n=0}^{\infty} Q_{n} \frac{x^{n}}{n !}-\frac{2}{b(a b+4)} \sum_{n=0}^{\infty} Q Q_{n} \frac{x^{n}}{n !}+\frac{a-b}{b(a b+4)} \\
= & \frac{1}{b(a b+4)}\left(\sum_{n=0}^{\infty}\left(Q_{n}-2 Q Q_{n}\right) \frac{x^{n}}{n !}+(a-b)\right) .
\end{aligned}
$$

Since

$$
f(x)^{2}=\sum_{n=0}^{\infty} \sum_{k=0}^{n}\left(\begin{array}{l}
n \\
k
\end{array}\right) q_{k} q_{n-k} \frac{x^{n}}{n !},
$$

by comparing the coefficients on both sides, we get the desired result.

Examples. For $n=1,2,3,4$ in Theorem 2.5, both sides of (2.7) equal to 0, 2, 6a, $6 a^{2}+8 a b+8$, respectively. 


\section{Higher-order identities}

Next, we shall consider the convolution identities for three bi-periodic Fibonacci numbers

$$
\sum_{\substack{k_{1}+k_{2}+k_{3}=n \\
k_{1}, k_{2}, k_{3} \geq 0}}\left(\begin{array}{c}
n \\
k_{1}, k_{2}, k_{3}
\end{array}\right) q_{k_{1}} q_{k_{2}} q_{k_{3}} .
$$

We use the following symmetric formula.

Lemma 3.1 ([17]). The following equality holds:

$$
\begin{aligned}
(\hat{a}+\hat{b}+\hat{c}+\hat{d})^{3} & \\
= & A\left(\hat{a}^{3}+\hat{b}^{3}+\hat{c}^{3}+\hat{d}^{3}\right)+B(\hat{a} \hat{b} \hat{c}+\hat{a} \hat{b} \hat{d}+\hat{a} \hat{c} \hat{d}+\hat{b} \hat{c} \hat{d}) \\
& +C\left(\hat{a}^{2}+\hat{b}^{2}+\hat{c}^{2}+\hat{d}^{2}\right)(\hat{a}+\hat{b}+\hat{c}+\hat{d}) \\
& +D(\hat{a} \hat{b}+\hat{a} \hat{c}+\hat{a} \hat{d}+\hat{b} \hat{c}+\hat{b} \hat{d}+\hat{c} \hat{d})(\hat{a}+\hat{b}+\hat{c}+\hat{d}),
\end{aligned}
$$

where $A=D-2, B=-3 D+6$ and $C=-D+3$.

\section{Lemma 3.2.}

$$
r_{1}^{3} e^{3 \alpha x}+r_{2}^{3} e^{-3 \alpha x}+r_{3}^{3} e^{3 \beta x}+r_{4}^{3} e^{-3 \beta x}=\frac{1}{4 b(a b+4)} \sum_{n=0}^{\infty} P_{n} \frac{x^{n}}{n !},
$$

where the numbers $P_{n}$ satisfy for $n \geq 1$

$$
\begin{aligned}
P_{2 n} & =\frac{3 a(a+3 b)}{3 a+b} P_{2 n-1}+9 P_{2 n-2}, \\
P_{2 n+1} & =\frac{3 b(3 a+b)}{a+3 b} P_{2 n}+9 P_{2 n-1}
\end{aligned}
$$

with $P_{0}=0$ and $P_{1}=3(3 a+b)$.

Remark 3.3. We have explicit expressions: for $n \geq 0$

$$
\begin{aligned}
P_{2 n} & =3^{2 n} a(a+3 b) \sum_{k=0}^{n-1}\left(\begin{array}{c}
2 n-k-1 \\
k
\end{array}\right)(a b)^{n-k-1}=3^{2 n}(a+3 b) q_{2 n}, \\
P_{2 n+1} & =3^{2 n+1}(3 a+b) \sum_{k=0}^{n}\left(\begin{array}{c}
2 n-k \\
k
\end{array}\right)(a b)^{n-k}=3^{2 n+1}(3 a+b) q_{2 n+1} .
\end{aligned}
$$

Proof of Lemma 3.2. Assume that the exponential generating function

$$
r_{1}^{3} e^{3 \alpha x}+r_{2}^{3} e^{-3 \alpha x}+r_{3}^{3} e^{3 \beta x}+r_{4}^{3} e^{-3 \beta x}
$$

determines the sequence $\left\{\widehat{P}_{n}\right\}_{n=0}^{\infty}$. By $\alpha \beta=1$ and $\alpha^{2}+\beta^{2}=a b+2$, we have the characteristic equation

$$
\begin{aligned}
& (x+3 \alpha)(x-3 \alpha)(x+3 \beta)(x-3 \beta) \\
& =x^{4}-9\left(\alpha^{2}+\beta^{2}\right) x^{2}+81 \alpha^{2} \beta^{2} \\
& =x^{4}-9(a b+2) x^{2}+81 .
\end{aligned}
$$

Thus, $\widehat{P}_{n}$ satisfies the recurrence relation

$$
\widehat{P}_{n}=9(a b+2) \widehat{P}_{n-2}-81 \widehat{P}_{n-4} \quad(n \geq 4) .
$$


Now, we get that

$$
\begin{aligned}
& \widehat{P}_{0}=r_{1}^{3}+r_{2}^{3}+r_{3}^{3}+r_{4}^{3}=0, \\
& \widehat{P}_{1}=3 r_{1}^{3} \alpha-3 r_{2}^{3} \alpha+3 r_{3}^{3} \beta-3 r_{4}^{3} \beta=\frac{3(3 a+b)}{4 b(a b+4)}, \\
& \widehat{P}_{2}=9 r_{1}^{3} \alpha^{2}+9 r_{2}^{3} \alpha^{2}+9 r_{3}^{3} \beta^{2}+9 r_{4}^{3} \beta^{2}=\frac{9 a(a+3 b)}{4 b(a b+4)}, \\
& \widehat{P}_{3}=27 r_{1}^{3} \alpha^{3}-27 r_{2}^{3} \alpha^{3}+27 r_{3}^{3} \beta^{3}-27 r_{4}^{3} \beta^{3}=\frac{27(3 a+b)(a b+1)}{4 b(a b+4)} .
\end{aligned}
$$

Using the recurrence relation (3.1), by induction, we have the recurrence relations: for $n \geq 1$

$$
\begin{aligned}
\widehat{P}_{2 n} & =\frac{3 a(a+3 b)}{3 a+b} \widehat{P}_{2 n-1}+9 \widehat{P}_{2 n-2}, \\
\widehat{P}_{2 n+1} & =\frac{3 b(3 a+b)}{a+3 b} \widehat{P}_{2 n}+9 \widehat{P}_{2 n-1} .
\end{aligned}
$$

Putting $P_{n}=4 b(a b+4) \widehat{P}_{n}$, we get the desired result.

\section{Lemma 3.4.}

$$
r_{1} r_{3} r_{4} e^{\alpha x}+r_{2} r_{3} r_{4} e^{-\alpha x}+r_{1} r_{2} r_{3} e^{\beta x}+r_{1} r_{2} r_{4} e^{-\beta x}=\frac{a-b}{4 b(a b+4)} \sum_{n=0}^{\infty} q_{n} \frac{x^{n}}{n !},
$$

Proof. Since

we get the result.

$$
r_{1} r_{2}=r_{3} r_{4}=\frac{a-b}{4 b(a b+4)}
$$

Theorem 3.5. For $n \geq 1$, we have

$$
\begin{aligned}
& \sum_{\substack{k_{1}+k_{2}+k_{3}=n \\
k_{1}, k_{2}, k_{3} \geq 0}}\left(\begin{array}{c}
n \\
k_{1}, k_{2}, k_{3}
\end{array}\right) q_{k_{1}} q_{k_{2}} q_{k_{3}} \\
= & \frac{1}{4 b(a b+4)}\left(A \cdot P_{n}+B(a-b) q_{n}+4 C \sum_{k=0}^{n}\left(\begin{array}{l}
n \\
k
\end{array}\right) Q_{k} q_{n-k}\right. \\
& \left.-4 D \sum_{k=0}^{n}\left(\begin{array}{l}
n \\
k
\end{array}\right) Q Q_{k} q_{n-k}+2(a-b) D q_{n}\right),
\end{aligned}
$$

where the numbers $A, B, C$ and $D$ satisfy the condition in Lemma 3.1.

Remark 3.6. It is clear that this value is 0 when $n=0,1,2$. Assume that $n \geq 3$. When $D=2$, by $A=B=0$ and $C=1$, we have a simpler form:

$$
\begin{aligned}
& \sum_{\substack{k_{1}+k_{2}+k_{3}=n \\
k_{1}, k_{2}, k_{3} \geq 0}}\left(\begin{array}{c}
n \\
k_{1}, k_{2}, k_{3}
\end{array}\right) q_{k_{1}} q_{k_{2}} q_{k_{3}} \\
= & \frac{1}{b(a b+4)}\left(\sum_{k=0}^{n}\left(\begin{array}{l}
n \\
k
\end{array}\right)\left(Q_{k}-2 Q Q_{k}\right) q_{n-k}+(a-b) q_{n}\right) \\
= & \frac{1}{b(a b+4)} \sum_{k=2}^{n-1}\left(\begin{array}{l}
n \\
k
\end{array}\right)\left(Q_{k}-2 Q Q_{k}\right) q_{n-k} .
\end{aligned}
$$

Notice that $Q_{0}-2 Q Q_{0}=-(a-b), Q_{1}-2 Q Q_{1}=0$ and $q_{0}=0$. 
Proof of Theorem 3.5. By Lemma 3.1 together with Lemmas 2.1, 2.3, 2.4, 3.2, 3.4, we have

$$
\begin{aligned}
&\left(r_{1} e^{\alpha x}+r_{2} e^{-\alpha x}+r_{3} e^{\beta x}+r_{4} e^{-\beta x}\right)^{3} \\
&=A\left(r_{1}^{3} e^{3 \alpha x}+r_{2}^{3} e^{-3 \alpha x}+r_{3}^{3} e^{3 \beta x}+r_{4}^{3} e^{-3 \beta x}\right) \\
&+ B\left(r_{1} r_{2} r_{3} e^{\beta x}+r_{1} r_{2} r_{4} e^{-\beta x}+r_{1} r_{3} r_{4} e^{\alpha x}+r_{2} r_{3} r_{4} e^{-\alpha x}\right) \\
&+C\left(r_{1}^{2} e^{2 \alpha x}+r_{2}^{2} e^{-2 \alpha x}+r_{3}^{2} e^{2 \beta x}+r_{4}^{2} e^{-2 \beta x}\right)\left(r_{1} e^{\alpha x}+r_{2} e^{-\alpha x}+r_{3} e^{\beta x}+r_{4} e^{-\beta x}\right) \\
&+D\left(r_{1} r_{2}+r_{1} r_{3} e^{(\alpha+\beta) x}+r_{1} r_{4} e^{(\alpha-\beta) x}+r_{2} r_{3} e^{-(\alpha-\beta) x}+r_{2} r_{4} e^{-(\alpha+\beta) x}+r_{3} r_{4}\right) \\
& \times\left(r_{1} e^{\alpha x}+r_{2} e^{-\alpha x}+r_{3} e^{\beta x}+r_{4} e^{-\beta x}\right) \\
&= A \frac{1}{4 b(a b+4)} \sum_{n=0}^{\infty} P_{n} \frac{x^{n}}{n !}+B \frac{a-b}{4 b(a b+4)} \sum_{n=0}^{\infty} q_{n} \frac{x^{n}}{n !} \\
&+C\left(\frac{1}{b(a b+4)} \sum_{n=0}^{\infty} Q_{n} \frac{x^{n}}{n !}\right)\left(\sum_{n=0}^{\infty} q_{n} \frac{x^{n}}{n !}\right) \\
&+D\left(-\frac{1}{b(a b+4)} \sum_{n=0}^{\infty} Q Q_{n} \frac{x^{n}}{n !}+\frac{a-b}{2 b(a b+4)}\right)\left(\sum_{n=0}^{\infty} q_{n} \frac{x^{n}}{n !}\right) \\
&= \frac{1}{4 b(a b+4)} \sum_{n=0}^{\infty}\left(A \cdot P_{n}+B(a-b) q_{n}+4 C \sum_{k=0}^{n}\left(\begin{array}{l}
n \\
k
\end{array}\right) Q_{k} q_{n-k}\right. \\
&\left.\quad-4 D \sum_{k=0}^{n}\left(\begin{array}{l}
n \\
k
\end{array}\right) Q Q_{k} q_{n-k}+2(a-b) D q_{n}\right) \frac{x^{n}}{n !} .
\end{aligned}
$$

On the other hand,

$$
\left(\sum_{n=0}^{\infty} q_{n} \frac{x^{n}}{n !}\right)^{3}=\sum_{n=0}^{\infty} \sum_{\substack{k_{1}+k_{2}+k_{3}=n \\
k_{1}, k_{2}, k_{3} \geq 0}}\left(\begin{array}{c}
n \\
k_{1}, k_{2}, k_{3}
\end{array}\right) q_{k_{1}} q_{k_{2}} q_{k_{3}} \frac{x^{n}}{n !} .
$$

Comparing the coefficients on both sides, we get the desired result.

Next, we shall consider the convolution identities for four bi-periodic Fibonacci numbers

$$
\sum_{\substack{k_{1}+k_{2}+k_{3}+k_{4}=n \\
k_{1}, k_{2}, k_{3}, k_{4} \geq 0}}\left(\begin{array}{c}
n \\
k_{1}, k_{2}, k_{3}, k_{4}
\end{array}\right) q_{k_{1}} q_{k_{2}} q_{k_{3}} q_{k_{4}} .
$$

We need the following symmetric formula.

Lemma 3.7 ([17]). The following equality holds:

$$
\begin{aligned}
(\hat{a}+ & \hat{b}+\hat{c}+\hat{d})^{4} \\
= & A\left(\hat{a}^{4}+\hat{b}^{4}+\hat{c}^{4}+\hat{d}^{4}\right)+B \hat{a} \hat{b} \hat{c} \hat{d}+C\left(\hat{a}^{3}+\hat{b}^{3}+\hat{c}^{3}+\hat{d}^{3}\right)(\hat{a}+\hat{b}+\hat{c}+\hat{d}) \\
& +D\left(\hat{a}^{2}+\hat{b}^{2}+\hat{c}^{2}+\hat{d}^{2}\right)^{2}+E\left(\hat{a}^{2}+\hat{b}^{2}+\hat{c}^{2}+\hat{d}^{2}\right)(\hat{a} \hat{b}+\hat{a} \hat{c}+\hat{a} \hat{d}+\hat{b} \hat{c}+\hat{b} \hat{d}+\hat{c} \hat{d}) \\
& +F(\hat{a} \hat{b}+\hat{a} \hat{c}+\hat{a} \hat{d}+\hat{b} \hat{c}+\hat{b} \hat{d}+\hat{c} \hat{d})^{2}+G\left(\hat{a}^{2}+\hat{b}^{2}+\hat{c}^{2}+\hat{d}^{2}\right)(\hat{a}+\hat{b}+\hat{c}+\hat{d})^{2} \\
& +H(\hat{a} \hat{b}+\hat{a} \hat{c}+\hat{a} \hat{d}+\hat{b} \hat{c}+\hat{b} \hat{d}+\hat{c} \hat{d})(\hat{a}+\hat{b}+\hat{c}+\hat{d})^{2} \\
& +I(\hat{a} \hat{b} \hat{c}(\hat{a}+\hat{b}+\hat{c})+\hat{a} \hat{b} \hat{d}(\hat{a}+\hat{b}+\hat{d})+\hat{b} \hat{c} \hat{d}(\hat{b}+\hat{c}+\hat{d})+\hat{a} \hat{c} \hat{d}(\hat{a}+\hat{c}+\hat{d})) \\
& +J(\hat{a} \hat{b} \hat{c}+\hat{a} \hat{b} \hat{d}+\hat{b} \hat{c} \hat{d}+\hat{a} \hat{c} \hat{d})(\hat{a}+\hat{b}+\hat{c}+\hat{d}),
\end{aligned}
$$

where $A=-D+E+G+H-3, B=12 D+12 G-4 J-12, C=-E-2 G-H+4$, $F=-2 D-2 G-2 H+6$ and $I=4 D-E+2 G-H-J$. 
Lemma 3.8.

$$
r_{1}^{4} e^{4 \alpha x}+r_{2}^{4} e^{-4 \alpha x}+r_{3}^{4} e^{4 \beta x}+r_{4}^{4} e^{-4 \beta x}=\frac{1}{4 b^{2}(a b+4)^{2}} \sum_{n=0}^{\infty} R_{n} \frac{x^{n}}{n !},
$$

where the numbers $R_{n}$ satisfy for $n \geq 1$

$$
\begin{aligned}
R_{2 n} & =\frac{a^{2}+6 a b+b^{2}}{a+b} R_{2 n-1}+2^{4} R_{2 n-2}, \\
R_{2 n+1} & =\frac{2^{4} a b(a+b)}{a^{2}+6 a b+b^{2}} R_{2 n}+2^{4} R_{2 n-1}
\end{aligned}
$$

with $R_{0}=a^{2}+6 a b+b^{2}$ and $R_{1}=8 a b(a+b)$.

Remark 3.9. We have explicit expressions: for $n \geq 0$

$$
\begin{aligned}
R_{2 n} & =2^{4 n-1}\left(a^{2}+6 a b+b^{2}\right) \sum_{k=0}^{n} \frac{2 n}{2 n-k}\left(\begin{array}{c}
2 n-k \\
k
\end{array}\right)(a b)^{n-k}, \\
P_{2 n+1} & =2^{4 n+3} a b(a+b) \sum_{k=0}^{n} \frac{2 n+1}{2 n-k+1}\left(\begin{array}{c}
2 n-k+1 \\
k
\end{array}\right)(a b)^{n-k} .
\end{aligned}
$$

Theorem 3.10. For $n \geq 1$

$$
\begin{aligned}
& \sum_{\substack{k_{1}+k_{2}+k_{3}+k_{4}=n \\
k_{1}, k_{2}, k_{3}, k_{4} \geq 0}}\left(\begin{array}{c}
n \\
k_{1}, k_{2}, k_{3}, k_{4}
\end{array}\right) q_{k_{1}} q_{k_{2}} q_{k_{3}} q_{k_{4}} \\
= & \frac{A}{4 b^{2}(a b+4)^{2}} R_{n}+\frac{C}{4 b(a b+4)} \sum_{k=0}^{n}\left(\begin{array}{l}
n \\
k
\end{array}\right) P_{k} q_{n-k}+\frac{D}{b^{2}(a b+4)^{2}} \sum_{k=0}^{n}\left(\begin{array}{l}
n \\
k
\end{array}\right) Q_{k} Q_{n-k} \\
& +E\left(\frac{1}{b^{2}(a b+4)^{2}} \sum_{k=0}^{n}\left(\begin{array}{l}
n \\
k
\end{array}\right) Q_{k} Q_{n-k}+\frac{a-b}{2 b^{2}(a b+4)^{2}} Q_{n}\right) \\
& +F\left(\frac{1}{b^{2}(a b+4)^{2}} \sum_{k=0}^{n}\left(\begin{array}{l}
n \\
k
\end{array}\right) Q Q_{k} Q Q_{n-k}+\frac{a-b}{b^{2}(a b+4)^{2}} Q Q_{n}\right) \\
& +\frac{G \quad n}{b(a b+4)} \sum_{\substack{k_{1}+k_{2}+k_{3}=n \\
k_{1}, k_{2}, k_{3} \geq 0}}\left(\begin{array}{c}
n \\
k_{1}, k_{2}, k_{3}
\end{array}\right) Q_{k_{1}} q_{k_{2}} q_{k_{3}} \\
& +H\left(\frac{1}{b(a b+4)} \sum_{\substack{k_{1}+k_{2}+k_{3}=n \\
k_{1}, k_{2}, k_{3} \geq 0}}\left(\begin{array}{c}
n \\
k_{1}, k_{2}, k_{3}
\end{array}\right) Q Q_{k_{1}} q_{k_{2}} q_{k_{3}}+\frac{a-b}{2 b(a b+4)}\left(\begin{array}{l}
n \\
k
\end{array}\right) Q_{k} Q_{n-k}\right) \\
& +I \frac{a-b}{4 b^{2}(a b+4)^{2}}\left(Q_{n}-Q Q_{n}\right)+J\left(\frac{1}{b(a b+4)} \sum_{k=0}^{n} Q Q_{k} q_{n-k}+\frac{a-b}{2 b(a b+4)} q_{n}\right)
\end{aligned}
$$

where the numbers $A, C, D, E, F, G, H, I$ and $J$ are given in Lemma 3.7.

Remark 3.11. The above form becomes much simpler for some specific values of the numbers $A$ to $J$. For example, when $A=C=G=H=I=0$, by $B=J=0, D=1$ and $E=F=4$, we have

$$
\begin{aligned}
& \sum_{\substack{k_{1}+k_{2}+k_{3}+k_{4}=n \\
k_{1}, k_{2}, k_{3}, k_{4} \geq 0}}\left(\begin{array}{c}
n \\
k_{1}, k_{2}, k_{3}, k_{4}
\end{array}\right) q_{k_{1}} q_{k_{2}} q_{k_{3}} q_{k_{4}} \\
= & \frac{1}{b^{2}(a b+4)^{2}}\left(2(a-b)\left(Q_{n}+2 Q Q_{n}\right)+\sum_{k=0}^{n}\left(\begin{array}{l}
n \\
k
\end{array}\right)\left(5 Q_{k} Q_{n-k}+4 Q Q_{k} Q Q_{n-k}\right)\right) .
\end{aligned}
$$


Proof of Theorem 3.10. We apply Lemma 3.7 as $\hat{a}=r_{1} e^{\alpha x}, \hat{b}=r_{2} e^{-\alpha x}, \hat{c}=r_{3} e^{\beta x}$ and $\hat{d}=r_{4} e^{-\beta x}$. By Lemma 3.8, we have

$$
A\left(\hat{a}^{4}+\hat{b}^{4}+\hat{c}^{4}+\hat{d}^{4}\right)=A \frac{1}{4 b^{2}(a b+4)^{2}} \sum_{n=0}^{\infty} R_{n} \frac{x^{n}}{n !} .
$$

By $r_{1} r_{2}=r_{3} r_{4}=B(a-b) / 4 b(a b+4)$,

$$
B \hat{a} \hat{b} \hat{c} \hat{d}=\frac{(a-b)^{2}}{16 b^{2}(a b+4)^{2}} .
$$

By Lemma 3.2,

$$
\begin{aligned}
& C\left(\hat{a}^{3}+\hat{b}^{3}+\hat{c}^{3}+\hat{d}^{3}\right)(\hat{a}+\hat{b}+\hat{c}+\hat{d}) \\
& =C\left(\frac{1}{4 b(a b+4)} \sum_{n=0}^{\infty} P_{n} \frac{x^{n}}{n !}\right)\left(\sum_{n=0}^{\infty} q_{n} \frac{x^{n}}{n !}\right) \\
& =C \frac{1}{4 b(a b+4)} \sum_{n=0}^{\infty} \sum_{k=0}^{n}\left(\begin{array}{l}
n \\
k
\end{array}\right) P_{k} q_{n-k} \frac{x^{n}}{n !} .
\end{aligned}
$$

By Lemma 2.1

$$
\begin{aligned}
& D\left(\hat{a}^{2}+\hat{b}^{2}+\hat{c}^{2}+\hat{d}^{2}\right)^{2} \\
& =D\left(\frac{1}{b(a b+4)} \sum_{n=0}^{\infty} Q_{n} \frac{x^{n}}{n !}\right)^{2} \\
& =D \frac{1}{b^{2}(a b+4)^{2}} \sum_{n=0}^{\infty} \sum_{k=0}^{n}\left(\begin{array}{l}
n \\
k
\end{array}\right) Q_{k} Q_{n-k} \frac{x^{n}}{n !} .
\end{aligned}
$$

By Lemma 2.3 and Lemma 2.4,

$$
\begin{aligned}
& E\left(\hat{a}^{2}+\hat{b}^{2}+\hat{c}^{2}+\hat{d}^{2}\right)(\hat{a} \hat{b}+\hat{a} \hat{c}+\hat{a} \hat{d}+\hat{b} \hat{c}+\hat{b} \hat{d}+\hat{c} \hat{d}) \\
& =E\left(\frac{1}{b(a b+4)} \sum_{n=0}^{\infty} Q_{n} \frac{x^{n}}{n !}\right)\left(\frac{1}{b(a b+4)} \sum_{n=0}^{\infty} Q Q_{n} \frac{x^{n}}{n !}+\frac{a-b}{2 b(a b+4)}\right) \\
& =E\left(\frac{1}{b^{2}(a b+4)^{2}} \sum_{n=0}^{\infty} \sum_{k=0}^{n}\left(\begin{array}{l}
n \\
k
\end{array}\right) Q_{k} Q_{n-k} \frac{x^{n}}{n !}+\frac{a-b}{2 b^{2}(a b+4)^{2}} \sum_{n=0}^{\infty} Q_{n} \frac{x^{n}}{n !}\right) .
\end{aligned}
$$

By Lemma 2.3 and Lemma 2.4,

$$
\begin{aligned}
& F(\hat{a} \hat{b}+\hat{a} \hat{c}+\hat{a} \hat{d}+\hat{b} \hat{c}+\hat{b} \hat{d}+\hat{c} \hat{d})^{2} \\
& =F\left(\frac{1}{b(a b+4)} \sum_{n=0}^{\infty} Q Q_{n} \frac{x^{n}}{n !}+\frac{a-b}{2 b(a b+4)}\right)^{2} \\
& =F\left(\frac{1}{b^{2}(a b+4)^{2}} \sum_{n=0}^{\infty} \sum_{k=0}^{n}\left(\begin{array}{l}
n \\
k
\end{array}\right) Q Q_{k} Q Q_{n-k} \frac{x^{n}}{n !}+\frac{a-b}{b^{2}(a b+4)^{2}} \sum_{n=0}^{\infty} Q Q_{n} \frac{x^{n}}{n !}+\frac{(a-b)^{2}}{4 b^{2}(a b+4)^{2}}\right) .
\end{aligned}
$$

By Lemma 2.1,

$$
\begin{aligned}
& G\left(\hat{a}^{2}+\hat{b}^{2}+\hat{c}^{2}+\hat{d}^{2}\right)(\hat{a}+\hat{b}+\hat{c}+\hat{d})^{2} \\
& =G\left(\frac{1}{b(a b+4)} \sum_{n=0}^{\infty} Q_{n} \frac{x^{n}}{n !}\right)\left(\sum_{n=0}^{\infty} q_{n} \frac{x^{n}}{n !}\right)^{2} \\
& =G \frac{1}{b(a b+4)} \sum_{n=0}^{\infty} \sum_{\substack{k_{1}+k_{2}+k_{3}=n \\
k_{1}, k_{2}, k_{3} \geq 0}}\left(\begin{array}{c}
n \\
k_{1}, k_{2}, k_{3}
\end{array}\right) Q_{k_{1}} q_{k_{2}} q_{k_{3}} \frac{x^{n}}{n !} .
\end{aligned}
$$


By Lemma 2.3 and Lemma 2.4,

$$
\begin{aligned}
& H(\hat{a} \hat{b}+\hat{a} \hat{c}+\hat{a} \hat{d}+\hat{b} \hat{c}+\hat{b} \hat{d}+\hat{c} \hat{d})(\hat{a}+\hat{b}+\hat{c}+\hat{d})^{2} \\
& =H\left(\frac{1}{b(a b+4)} \sum_{n=0}^{\infty} Q Q_{n} \frac{x^{n}}{n !}+\frac{a-b}{2 b(a b+4)}\right)\left(\sum_{n=0}^{\infty} q_{n} \frac{x^{n}}{n !}\right)^{2} \\
& =H\left(\frac{1}{b(a b+4)} \sum_{n=0}^{\infty} \sum_{\substack{k_{1}+k_{2}+k_{3}=n \\
k_{1}, k_{2}, k_{3} \geq 0}}\left(\begin{array}{c}
n \\
k_{1}, k_{2}, k_{3}
\end{array}\right) Q Q_{k_{1}} q_{k_{2}} q_{k_{3}} \frac{x^{n}}{n !}\right. \\
& \left.\quad+\frac{a-b}{2 b(a b+4)} \sum_{n=0}^{\infty} \sum_{k=0}^{n}\left(\begin{array}{l}
n \\
k
\end{array}\right) Q_{k} Q_{n-k} \frac{x^{n}}{n !}\right) .
\end{aligned}
$$

By Lemma 2.1 and Lemma 2.3 with $r_{1} r_{2}=r_{3} r_{4}=(a-b) / 4 b(a b+4)$,

$$
\begin{aligned}
I(\hat{a} \hat{b} \hat{c}(\hat{a}+\hat{b}+\hat{c})+\hat{a} \hat{b} \hat{d}(\hat{a}+\hat{b}+\hat{d})+\hat{b} \hat{c} \hat{d}(\hat{b}+\hat{c}+\hat{d})+\hat{a} \hat{c} \hat{d}(\hat{a}+\hat{c}+\hat{d})) \\
=I \frac{a-b}{4 b(a b+4)}\left(r_{1} r_{3} e^{(\alpha+\beta) x}+r_{2} r_{4} e^{-(\alpha+\beta) x}+r_{1} r_{4} e^{(\alpha-\beta) x}+r_{2} r_{3} e^{-(\alpha-\beta) x}\right) \\
\quad+I\left(r_{1}^{2} e^{2 \alpha x}+r_{2}^{2} e^{-2 \alpha x}+r_{3}^{2} e^{2 \beta x}+r_{4}^{2} e^{-2 \beta x}\right) \\
=-I \frac{a-b}{4 b(a b+4)} \frac{1}{b(a b+4)} \sum_{n=0}^{\infty} Q Q_{n} \frac{x^{n}}{n !}+I \frac{a-b}{4 b(a b+4)} \frac{1}{b(a b+4)} \sum_{n=0}^{\infty} Q_{n} \frac{x^{n}}{n !} \\
=I \frac{a-b}{4 b^{2}(a b+4)^{2}} \sum_{n=0}^{\infty}\left(Q_{n}-Q Q_{n}\right) \frac{x^{n}}{n !} .
\end{aligned}
$$

By Lemma 2.3 and Lemma 2.4,

$$
\begin{aligned}
& J(\hat{a} \hat{b} \hat{c}+\hat{a} \hat{b} \hat{d}+\hat{b} \hat{c} \hat{d}+\hat{a} \hat{c} \hat{d})(\hat{a}+\hat{b}+\hat{c}+\hat{d}) \\
& =J\left(\frac{1}{b(a b+4)} \sum_{n=0}^{\infty} Q Q_{n} \frac{x^{n}}{n !}+\frac{a-b}{2 b(a b+4)}\right)\left(\sum_{n=0}^{\infty} q_{n} \frac{x^{n}}{n !}\right) \\
& =J\left(\frac{1}{b(a b+4)} \sum_{n=0}^{\infty} \sum_{k=0}^{n} Q Q_{k} q_{n-k} \frac{x^{n}}{n !}+\frac{a-b}{2 b(a b+4)} \sum_{n=0}^{\infty} q_{n} \frac{x^{n}}{n !}\right) .
\end{aligned}
$$

We combine all the relations to get the main result.

\section{Final remarks}

One can continue to get the convolution identities of five and more bi-periodic Fibonacci numbers. The situation becomes more complicated. Even in the case of five bi-periodic Fibonacci numbers, we need the symmetric formula for $(\hat{a}+\hat{b}+\hat{c}+\hat{d})^{5}$ (see $\left.[12,17]\right)$.

Acknowledgment. The authors thank the anonymous referee for careful reading of the manuscript and helpful comments and suggestions. The research of José L. Ramírez was partially supported by Universidad Nacional de Colombia, Project No. 37805.

\section{References}

[1] T. Agoh and K. Dilcher, Convolution identities and lacunary recurrences for Bernoulli numbers, J. Number Theory, 124, 105-122, 2007.

[2] T. Agoh and K. Dilcher, Higher-order recurrences for Bernoulli numbers, J. Number Theory, 129, 1837-1847, 2009.

[3] T. Agoh and K. Dilcher, Higher-order convolutions for Bernoulli and Euler polynomials, J. Math. Anal. Appl. 419, 1235-1247, 2014. 
[4] M. Alp, N. Irmak and L. Szalay, Two-Periodic ternary recurrences and their Binetformula, Acta Math. Univ. Comenianae 2, 227-232, 2012.

[5] C. Cooper, Some identities involving differences of products of generalized Fibonacci numbers, Colloq. Math. 141 (1), 45-49, 2015.

[6] K. Dilcher and C. Vignat, General convolution identities for Bernoulli and Euler polynomials, J. Math. Anal. Appl. 435, 1478-1498, 2016.

[7] M. Edson and O. Yayenie, A new generalization of Fibonacci sequences and extended Binet's Formula, Integers, 9 (A48), 639-654, 2009.

[8] N. Irmak and L. Szalay, On k-periodic binary recurrences, Ann. Math. Inform. 40, 25-35, 2012.

[9] T. Komatsu, Higher-order convolution identities for Cauchy numbers of the second kind, Proc. Jangjeon Math. Soc. 18, 369-383, 2015.

[10] T. Komatsu, Higher-order convolution identities for Cauchy numbers, Tokyo J. Math. 39, 225-239, 2016.

[11] T. Komatsu, Convolution identities for Tribonacci numbers, Ars Combin. 136, 199$210,2018$.

[12] T. Komatsu and R. Li, Convolution identities for Tribonacci numbers with symmetric formulae, Math. Rep. (Bucur.) 21 (1), 27-47, 2019, arXiv:1610.02559.

[13] T. Komatsu, Z. Masakova and E. Pelantova, Higher-order identities for Fibonacci numbers, Fibonacci Quart. 52 (5), 150-163, 2014.

[14] T. Komatsu and G.K. Panda, On several kinds of sums involving balancing and Lucasbalancing numbers, Ars Combin. (to appear). arXiv:1608.05918.

[15] T. Komatsu and P.K. Ray, Higher-order identities for balancing numbers, arXiv:1608.05925, 2016.

[16] T. Komatsu and Y. Simsek, Third and higher order convolution identities for Cauchy numbers, Filomat 30, 1053-1060, 2016.

[17] R. Li, Convolution identities for Tetranacci numbers, arXiv:1609.05272.

[18] J.L. Ramírez, Bi-periodic incomplete Fibonacci sequences, Ann. Math. Inform. 42, 83-92, 2013.

[19] W. Wang, Some results on sums of products of Bernoulli polynomials and Euler polynomials, Ramanujan J. 32, 159-186, 2013.

[20] O. Yayenie, A note on generalized Fibonacci sequence, Applied. Math. Comp. 217 (12), 5603-5611, 2011. 